\title{
Accidentally-damaged REBOA catheter in a trauma patient: a case report
}

Received April 15, 2020

Revised June 11, 2020

Accepted June 11, 2020

Correspondence to

Pil Young Jung

Department of Surgery, Yonsei

University Wonju College of Medicine,

20 Ilsan-ro, Wonju 26426, Korea

Tel: +82-33-741-0882

Fax: +82-33-741-0574

E-mail: surgery4trauma@yonsei.ac.kr

\author{
Gaesung Ha, Seongyup Kim, Pil Young Jung \\ Department of Surgery, Yonsei University Wonju College of Medicine, Wonju Severance \\ Christian Hospital, Wonju, Korea
}

For a hemodynamically unstable patient with massive hemorrhage, resuscitative endovascular balloon occlusion of the aorta (REBOA) can be an option, as its use provides valuable time to find and repair the site of bleeding. This procedure appears less invasive through an endovascular approach and is relatively easy to perform. We describe an uncommon complication wherein the REBOA catheter was broken during its removal.

Key Words: Balloon occlusion, Aorta, Endovascular procedure

\section{Introduction}

Resuscitative endovascular balloon occlusion of the aorta (REBOA) is an effective treatment option in hemodynamically unstable patients with uncontrolled hemorrhage, promoting temporary stability during injury repair. This procedure appears to be less invasive and relatively easy to perform, even for non-surgeons. However, it is a recently developed technique and may entail some uncommon complications. We report an incident of balloon catheter tip breakage during REBOA catheter removal.

\section{Case presentation}

A 66-year-old male patient, with an unremarkable past medical history, was admitted via the emergency department with blunt trauma. He presented in shock with an open wound of his left thigh and massive bleeding, suggestive of left femoral vessel injury, along with a traumatic intracerebral hemorrhage, a left femoral mid-shaft frac- ture and left hip dislocation. His initial blood pressure was 50/20mm Hg and pulse rate was 130/minute. Our trauma team decided to perform REBOA using a RESCUE balloon $^{\text {TM }}$ catheter (Tokai Medical Products, Aichi, Japan) and 7 Fr. Radiofocus ${ }^{\circledR}$ Introducer II (Terumo, Tokyo, Japan) via his right common femoral artery, targeting zone III. After performing total inflation of the balloon using normal saline, we checked its position using portable X-ray, and found that the catheter was placed at zone II (Fig. 1). We planned to reposition the REBOA catheter to zone III in the emergency room, however, the patient's vital signs worsened and we decided to reposition the catheter in the operating room without further imaging. The patient was sent to the operating room with the REBOA catheter, 20 minutes post-arrival at the emergency room. During the operation, partial rupture of the left femoral vein was revealed (Fig. 2) and venorrhaphy of the femoral vein was performed. During the operation, we partially deflated the balloon and found the bleeding source in the left femoral vein; we immediately reinflated the balloon and repaired

(c)This is an Open Access article distributed under the terms of the Creative Commons Attribution Non-Commercial License (http://creativecommons.org/licenses/by-nc/4.0) which permits unrestricted noncommercial use, distribution, and reproduction in any medium, provided the original work is properly cited.

Copyright (C) 2020 Korean Association for Research, Procedures and Education on Trauma. All rights reserved. 
the vessel. After the venorrhaphy, we partially deflated the balloon again in order to confirm that there was no further bleeding from the repaired vein. No further bleeding was occurring, and the patient's vital signs stabilized, therefore we did not need to inflate the balloon again. Following the venorrhaphy, external fixation of the femoral fracture and closed reduction of the hip dislocation were performed by an orthopedic surgeon. Total occlusion time was 54 minutes, excluding the deflation and re-inflation time spent to check for the bleeding site and post-venorrhaphy status, which lasted for approximately three minutes each. Nearly ten hours after the operation, we removed the REBOA catheter in the intensive care unit. We tried to remove the REBOA catheter without the sheath, but the catheter tip with the balloon was broken during the removal procedure, despite not using much force. As a result, the broken

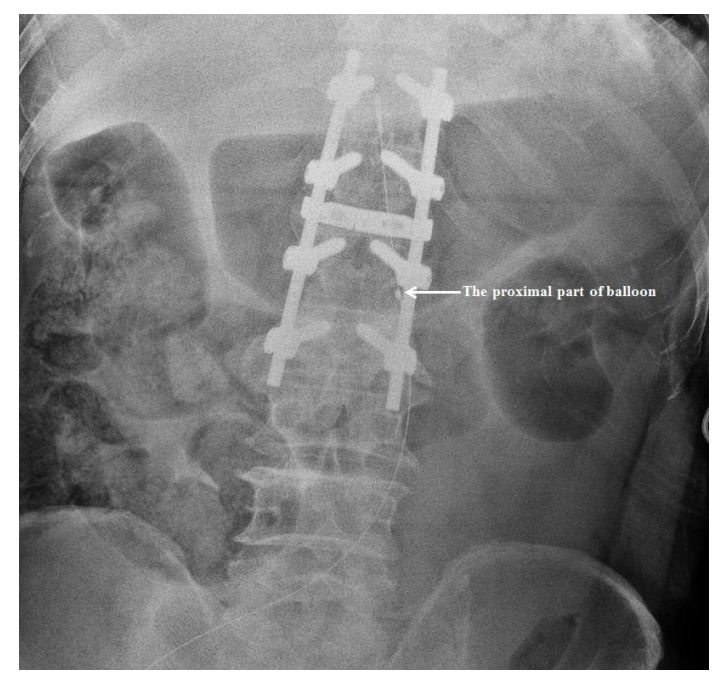

Fig. 1. Portable abdomen flat X-ray following REBOA depicting balloon position in Zone II. fragment remained in the subcutaneous tissue with its tip through the skin layer. After dissecting through a small skin incision, we successfully removed the remnant catheter and its sheath without further complication (Fig. 3A and $3 \mathrm{~B})$.

\section{Discussion}

In an urgent setting of massive bleeding from a traumatic major vessel injury, the REBOA procedure is commonly used as a bridging technique $(1,2)$. It maintains blood pressure from reaching a shock level, providing an opportunity to stop the hemorrhage (3). Following the ballooning procedure, the shape of the catheter can change such that it becomes difficult to remove. In our case, we initially attempted to remove the balloon catheter without its sheath in order to minimize vessel injury, as the irregular shape of the deflated catheter can damage the vessel wall. In order to prevent the catheter-breaking event that occurred with our patient, the user's guide of the RESCUE balloon ${ }^{\mathrm{TM}}$ catheter recommends removing the

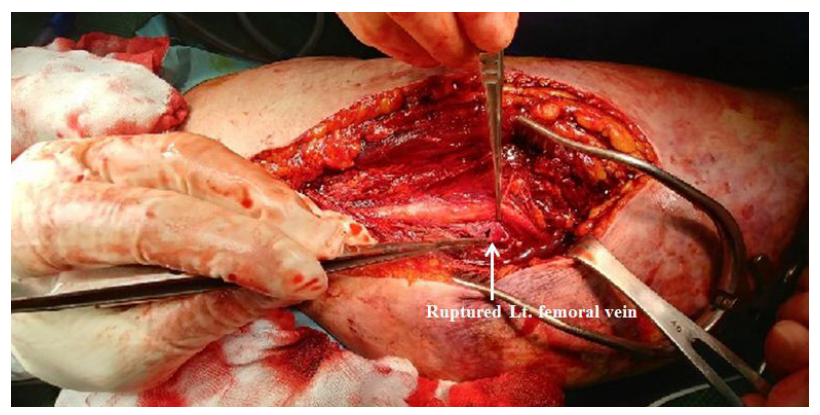

Fig. 2. Partially ruptured left femoral vein observed during surgery.
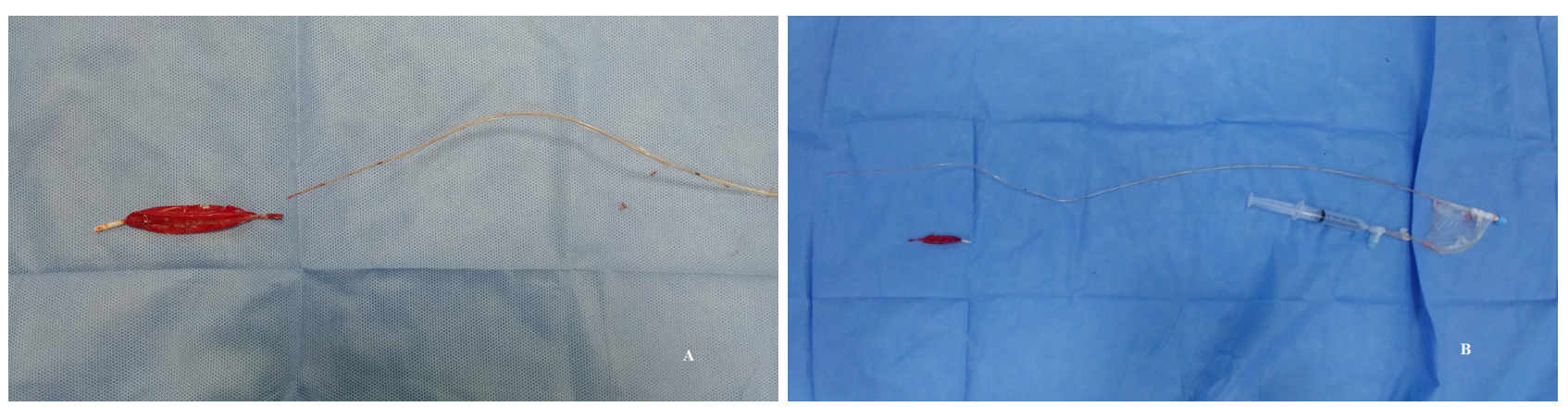

Fig. 3. Broken REBOA catheter; (A) Close view of the connecting portion with balloon after removal procedure, (B) Whole REBOA catheter that was broken. 
sheath and balloon catheter at the same time. Therefore, simultaneous removal of the balloon catheter and sheath should have been considered. There is also the possibility of sheath tip injury when insertion is performed without a skin incision. As another cause of the balloon catheter breakage, it is possible that the balloon was not completely deflated. With traction, the partially inflated balloon may have caused resistance against the traction force, resulting in catheter breakage. Total deflation should always be confirmed prior to removing the balloon catheter. However, in this case, we initially made a skin incision, followed by the routine maneuver of priming and total deflation, and the accidental event occurred. To prevent a catheter-breaking accident, we could consider using a larger sheath $(>7 \mathrm{Fr}$.) in order to reduce the traction resistance, and if resistance is present, consider removing the balloon catheter along with the sheath simultaneously. Conclusively we should consider an unexpected accident in a routine REBOA procedure.

\section{Conflict of interest}

No potential conflict of interest relevant to this article was reported.

\section{References}

1. Magee GA, Fox CJ, Moore EE. Resuscitative endovascular balloon occlusion of the aorta in pelvic ring fractures: The Denver Health protocol. Injury. 2020; S0020-1383(20)30072-3.

2. Pieper A, Thony F, Brun J, Rodière $M$, Boussat $B$, Arvieux C, et al. Resuscitative endovascular balloon occlusion of the aorta for pelvic blunt trauma and life-threatening hemorrhage: a 20 -year experience in a level I trauma center. J. Trauma Acute Care Surg. 2018;84(3):449-53.

3. Özkurtul O, Staab H, Osterhoff G, Ondruschka B, Höch A, Josten C, et al. Technical limitations of RE$\mathrm{BOA}$ in a patient with exsanguinating pelvic crush trauma: a case report. Patient Saf Surg. 2019;13(1):25. 